\title{
Anquilosis temporomandibular en pacientes pediátricos
}

\author{
Temporomandibular joint ankylosis in pediatric patients
}

\author{
Víctor Daniel Cázarez-Ríos, ${ }^{*+}$ Jorge Téllez-Rodríguez, ${ }^{\ddagger}$ Rubí López-Fernández ${ }^{\S}$ \\ * Residente de cuarto año de Cirugía Maxilofacial, Hospital Regional “Lic. Adolfo López Mateos”, ISSSTE. ‡ Cirujano Maxilofacial \\ Jefe de Servicio de Estomatología Pediátrica, Instituto Nacional de Pediatría. ${ }^{\S}$ Cirujano Maxilofacial Médico adscrito al Servicio de \\ Estomatología Pediátrica, Instituto Nacional de Pediatría, Ciudad de México, México.
}

\begin{abstract}
RESUMEN
La anquilosis de la articulación temporomandibular en pacientes pediátricos representa un reto, puesto que es una patología compleja. La etiología más frecuente es por traumatismo mandibular. Esta condición limita la función masticatoria y del habla, pudiendo llevar a problemas psicosociales del paciente. El tratamiento puede ser mediante injerto autólogo, prótesis articular o distracción osteogénica. Se presenta el caso de un paciente de 10 años de edad, en quien el tratamiento quirúrgico fue exitoso, mediante la eliminación de la anquilosis y la colocación de injerto costocondral.
\end{abstract}

Palabras clave: Anquilosis, articulación temporomandibular, injerto costocondral.

\section{INTRODUCCIÓN}

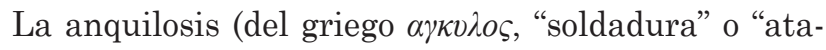
dura") de la articulación temporomandibular (ATM) se clasifica como un trastorno de hipomovilidad crónica, que lleva a limitación parcial o total de los movimientos mandibulares..$^{1-3}$ Esta condición interfiere con el habla, masticación e higiene, pero además altera el desarrollo y crecimiento maxilofacial, asimismo, puede llevar a

\footnotetext{
+Correspondencia: VDCR, victor_dl2@hotmail.com

Conflicto de intereses: Los autores declaran que no tienen.

Citar como: Cázarez-Ríos VD, Téllez-Rodríguez J, LópezFernández R. Anquilosis temporomandibular en pacientes pediátricos. Rev Mex Pediatr. 2020; 87(5): 189-194. https://dx.doi. org/10.35366/97173
}

\begin{abstract}
Ankylosis temporomandibular joint in pediatric patients is a challenge for the oral surgeon, since it is a complex disease. The most common cause is trauma mandibular. This condition limits the chewing and speech function, which can lead to psychosocial problems. Treatment can be by autologous graft, joint prosthesis, or osteogenic distraction.

We present the case of a 10-year-old patient, in whom the successful treatment of temporomandibular ankylosis was surgical, through the removal of the ankylosis and the placement of a costochondral graft.
\end{abstract}

Keywords: Ankylosis, temporomandibular joint, costochondral graft.

desnutrición y repercusiones psicosociales. Además, existe el riesgo de ser una emergencia médica cuando hay compromiso de vía aérea. ${ }^{4}$

Existen pocos datos sobre la epidemiología de anquilosis temporomandibular. Se ha reportado que entre 83 y $98.5 \%$ es de origen traumático, mientras que ocurre en niños en una frecuencia de 41 a $59.5 \%$, existe predilección al sexo femenino (52\%), siendo bilateral en $54 \%$. En el $88 \%$ existe anquilosis ósea y en $45 \%$ es del tipo III, según Shawney. ${ }^{3}$

La causa más común se debe a hemartrosis secundaria a traumatismo, ${ }^{3-8}$ pero también puede ser de causas autoinmunes, infecciosas o congénitas. ${ }^{7} \mathrm{La}$ hemartrosis ocasiona adherencias articulares, lo cual gradualmente ocasiona limitación significativa de la cinemática mandibular, que lleva a más adherencias 
hasta llegar a la anquilosis. En algunos casos, el traumatismo original produce desplazamiento del periostio, ocurriendo una unión directa del cóndilo con la ATM. ${ }^{3}$ Se considera una verdadera anquilosis cualquier condición que genere una adhesión ósea o fibrosa entre las superficies de la ATM.

\section{PRESENTACIÓN DEL CASO}

Presentamos el caso de un paciente masculino de 10 años de edad, quien acude al Instituto Nacional de Pediatría de México por presentar asimetría facial, con antecedente de trauma en la región mandibular a los tres años de edad. La asimetría fue progresiva hasta la limitación a la apertura bucal posterior a los seis años de edad.

En la valoración clínica inicial presentaba apertura oral limitada a $12 \mathrm{~mm}$, oclusión estable, canteamiento maxilar y dentición secundaria incompleta.
Se realizó estudio tomográfico, en el cual se observa masa anquilótica en zona de ATM del lado derecho e hiperplasia coronoidea ipsilateral.

Se decidió realizar escisión de masa anquilótica y coronoidectomía, con toma y colocación de injerto costocondral para reconstrucción de complejo temporomandibular derecho (Figura 1). Mediante estereolitografía se planeó el procedimiento quirúrgico (Figura 2).

La cirugía se realizó con anestesia general balanceada. El abordaje submandibular del lado derecho consistió en la disección subperióstica hasta alcanzar la anquilosis y proceso coronoideo. Se marca zona de osteotomía para segmentar la anquilosis y proceso coronoideo, y mediante sierras oscilante y reciprocante se osteotomiza y se concluye con escisión con cinceles, logrando una apertura oral inmediata de 32 mm (Figura 3).

Se procede a la toma de injerto costocondral de séptimo arco costal izquierdo, obteniendo $3 \mathrm{~cm}$ de
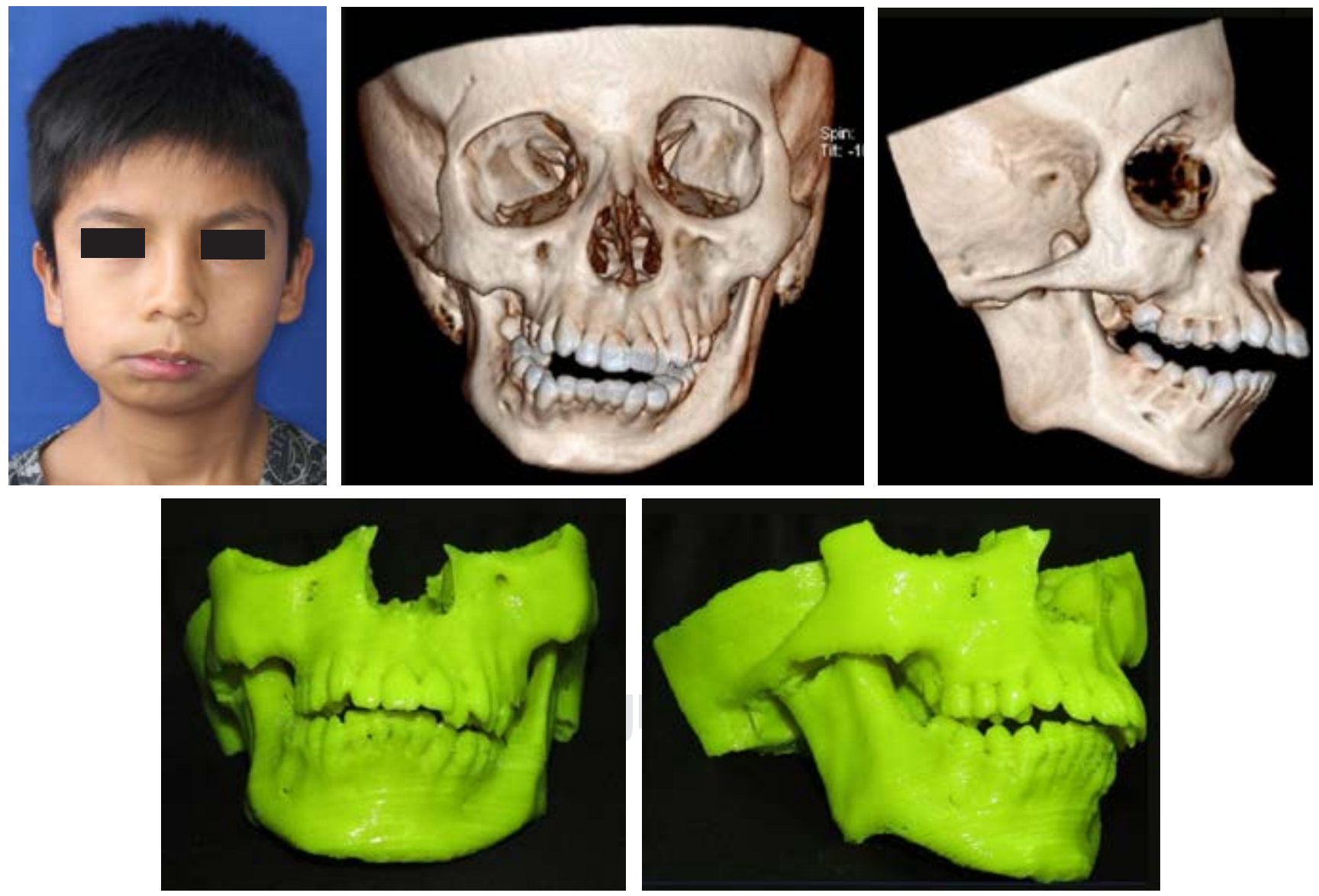

Figura 1: Vista clínica e imagenológica. 

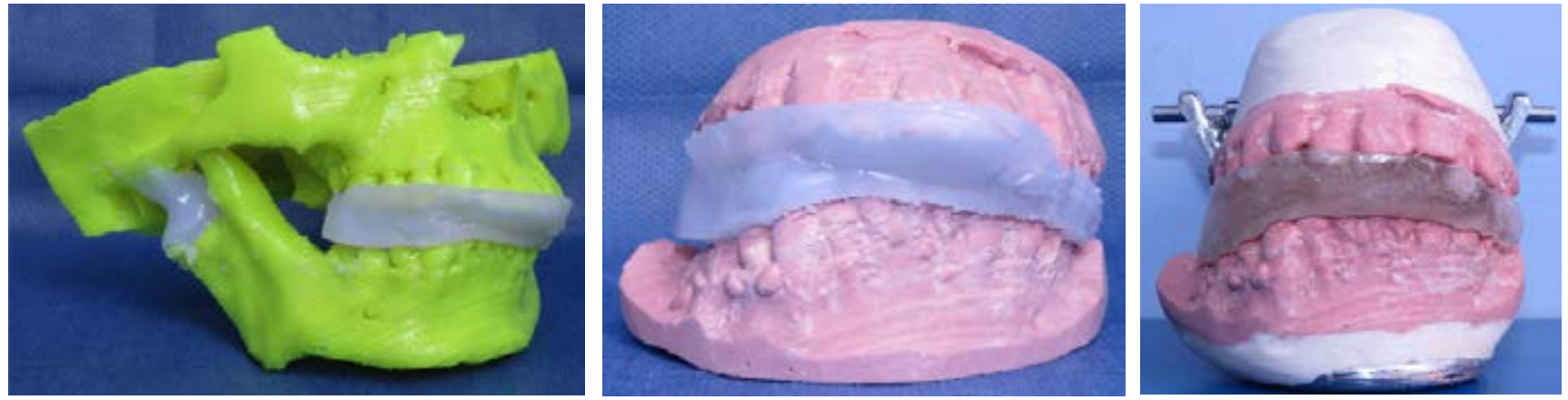

Figura 2: Preparación de guía quirúrgica.
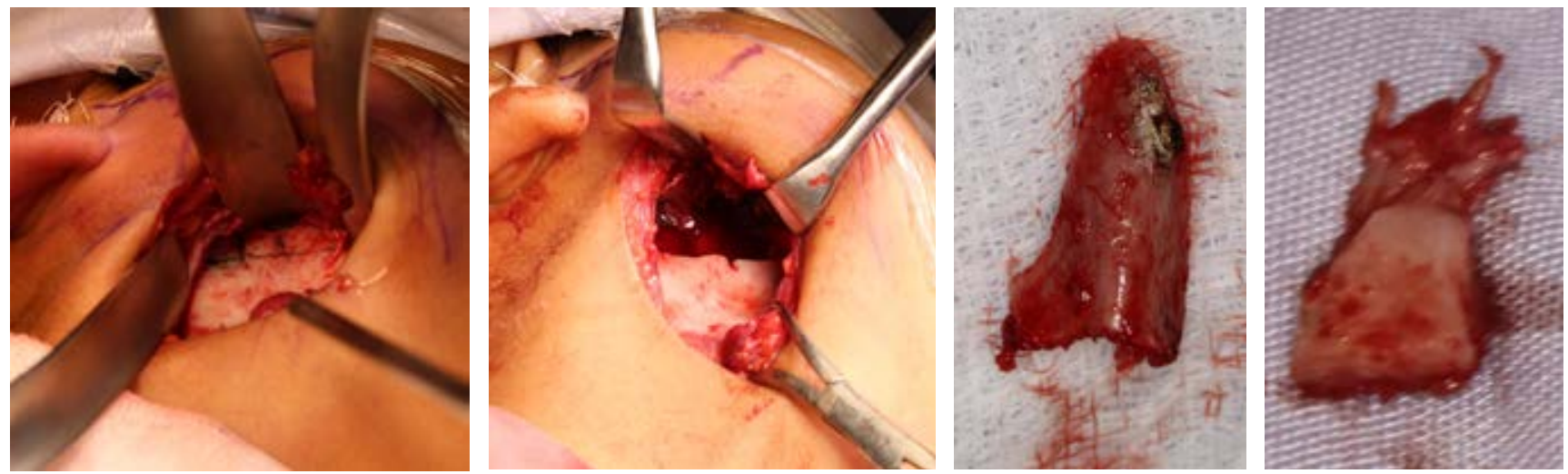

Figura 3: Escisión de la masa anquilosada y coronoides.

injerto óseo y $1 \mathrm{~cm}$ de cabeza cartilaginosa libres de periostio, los cuales fueron confeccionados anatómicamente; además se tomaron $2 \mathrm{~cm}^{3}$ de tejido adiposo para interposición en injerto y zona receptora (Figura 4). Posteriormente, se realiza sinéresis de abordaje por planos anatómicos mediante puntos de sutura de ácido poliglicólico 3-0. El injerto se lleva a zona de rama mandibular derecha, se coloca guía quirúrgica preconformada y, mediante tracción inferior de rama mandibular, se estabiliza y fija con tres tornillos bicorticales de titanio, buscando adecuada adaptación a zona articular. Se verificó cinemática mandibular, la cual permite $32 \mathrm{~mm}$ (Figura 5), considerándose adecuada. Finalmente se realizó sinéresis de abordaje mediante puntos simples invertidos para planos anatómicos subcutáneos en abordaje submandibular y piel. Posteriormente se realiza estudio tomográfico de control para valorar la adecuada posición del injerto (Figura 6).

Después de la cirugía, se inicia fisioterapia enérgica de apertura oral para evitar reanquilosis del tejido.
Se inicia protocolo de terapia funcional ${ }^{9}$ mediante ejercicios de apertura oral en las primeras 24 horas con abrebocas tipo Molt, cuatro veces al día. Pasada la primera semana, se colocó aparato ortopédico funcional con Spring Bite-Woodside, para favorecer la autorrotación anterior de la mandíbula.

El paciente se mantendrá en seguimiento para valorar evolución, así como la necesidad de cirugía ortognática debido a la deformidad dentofacial que ocasionó la anquilosis (Figura 6).

\section{DISCUSIÓN}

Existen diversas clasificaciones de la anquilosis temporomandibular (Rowe, Topazian, Shawney, siendo esta última de las más utilizadas actualmente) las cuales dependen de diversos factores: ${ }^{1-8,10,11}$

1. Por la localización del tejido anquilosado: intra-, extra- o yuxtaarticular.

2. Por las articulaciones afectadas: unilateral o bilateral. 
3. Por su extensión: parcial o total.

4. Por el grado de movilidad que permite: hipomóvil o inmóvil.

5. Por el tipo de tejido predominante: fibrosa, ósea o fibro-ósea.

6. Por la etapa en la que se presente: congénita (es muy rara y grave, ya que la cavidad glenoidea, el cóndilo mandibular y el proceso coronoideo forman un monobloque óseo) o adquirida.

Shawney la clasifica en cuatro grados: ${ }^{8}$

1. Cabeza condilar presente pero deforme, las adhesiones fibrosas limitan la movilidad.

2. Fusión ósea entre la cabeza condilar deforme y la cavidad glenoidea, particularmente entre los aspectos anterior, lateral y posterior, la parte medial permanece inalterada.

3. Bloque óseo entre la rama mandibular y el arco zigomático, todavía puede ser visible la cabeza con- dilar atrófica y desplazada hacia la parte medial, el disco articular puede estar intacto en el aspecto más profundo

4. La anatomía de la ATM está completamente perdida y existe un bloque óseo franco entre la rama y la base de cráneo.

El tratamiento, como en el presente caso, es quirúrgico y tiene como objetivo la liberación de la anquilosis mandibular. ${ }^{7}$ El estándar de oro para esta patología es la remoción quirúrgica de la masa anquilosada, lo cual se complementa con el uso de injertos autólogos (en niños se prefiere el costocondral) para la reconstrucción de la ATM. ${ }^{2}$ Existen alternativas, como la artroplastia, prótesis articulares o distracción osteogénica, cada una de ellas con ciertas ventajas y complicaciones asociadas.

La colocación del injerto (costocondral) permite buena adaptabilidad en la zona receptora y especialmente la incorporación de una cabeza cartilaginosa a la zona articular, lo que reduce la posibilidad de reanquilosis,
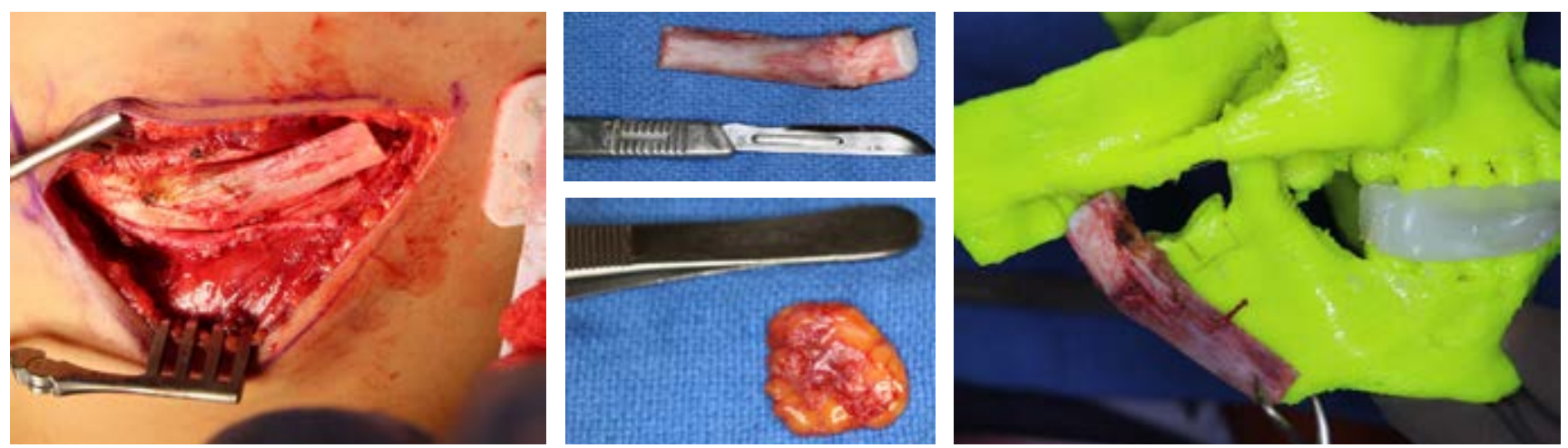

Figura 4: Injerto costocondral y de tejido adiposo.
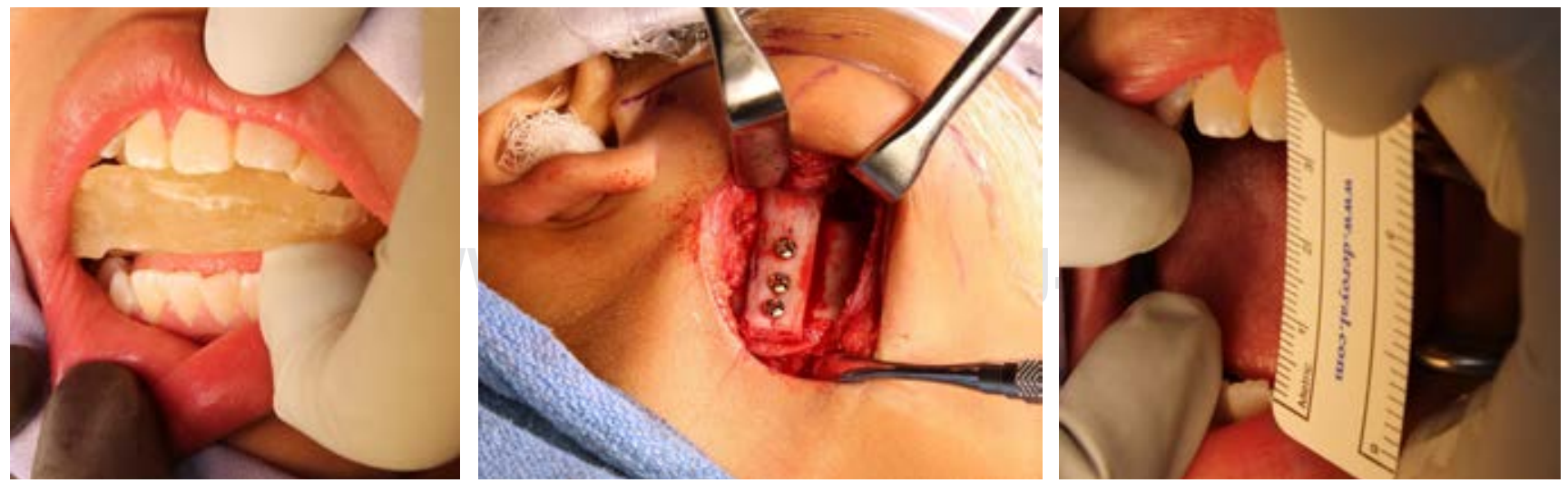

Figura 5: Colocación de injerto costal y apertura oral. 

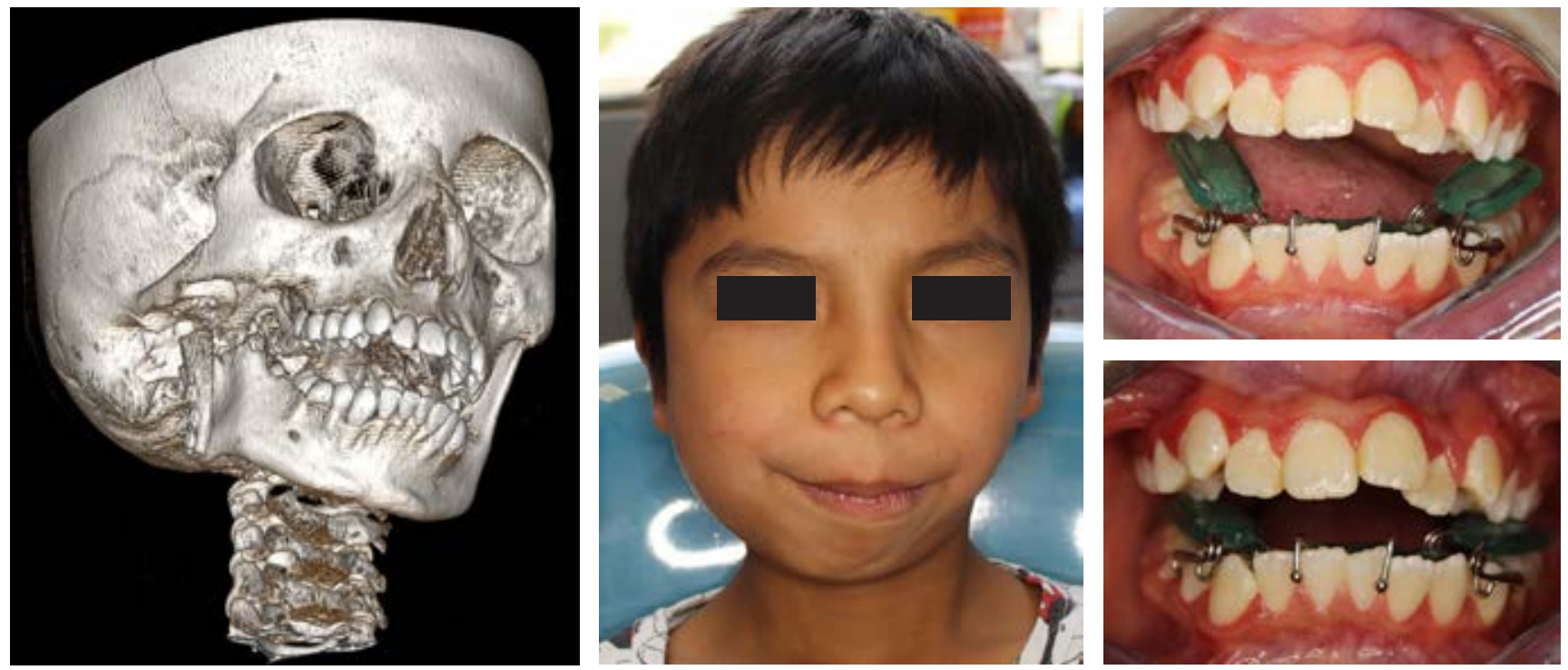

Figura 6: Tomografía de control y aparatología ortopédica.

pocos casos han reportado el crecimiento del injerto (hasta 54\%) asociada a la porción de tejido cartilaginoso, ${ }^{2,3,10,12}$ lo cual interfiere con la dimensión planeada. ${ }^{2}$ Para reducir la posibilidad de reanquilosis, se incluye tejido de interposición como músculo temporal, cartílago, fascia, grasa, dermis o piel total.

En cuanto a la fijación, puede ser con miniplacas, con tornillos bicorticales o fijación aislada con los mismos. ${ }^{1-8,10}$ Se ha descrito la fractura en tallo verde del injerto para mejorar la adaptación a la zona articular; en ocasiones se ha reportado un restablecimiento total de la zona articular temporomandibular. Belmont y cols. en 2007 propusieron una modificación en el protocolo de Kaban, en el cual se agrega aparato ortopédico dental, continuando de esta manera con las recomendaciones de terapia enérgica, que se inicia lo más rápido posible tras la cirugía. ${ }^{9}$

La recurrencia de la anquilosis representa el mayor problema, por lo que es esencial la remoción amplia del tejido anquilótico; se recomienda realizar artroplastia para mantener una dimensión de al menos $15 \mathrm{~mm}$ entre la fosa glenoidea reconformada y la mandíbula. Además, deberán indicarse ejercicios y fisioterapia para mejorar la función. En la actualidad, algunos cirujanos plantean que es necesaria la interposición de un material en el espacio creado para prevenir la recurrencia, prefiriéndose desde el injerto graso, para evitar formación de tejido, ${ }^{3}$ hasta materiales aloplásticos; el objetivo es devolver la máxima funcionalidad y evitar la recurrencia. ${ }^{8}$
En niños, esperar que el tratamiento se realice hasta el desarrollo total del macizo facial no es una indicación para el reemplazo de la ATM, debido a la alta posibilidad de presentar asimetrías de manera secundaria, elongación o hipertrofia del proceso coronoideo, limitando aún más la apertura bucal. ${ }^{13} \mathrm{El}$ pronóstico de un progreso favorable con tratamiento es inversamente proporcional al paso de años que se retrase el tratamiento.

El protocolo quirúrgico descrito por Kaban para anquilosis de la ATM en niños consta de siete pasos:

1. Escisión agresiva de la masa ósea o fibrosa.

2. Coronoidectomía en el lado afectado.

3. Coronoidectomía en el lado contralateral si los pasos 1 y 2 no favorecieron más de $35 \mathrm{~mm}$ de apertura o al punto de luxación del lado contrario.

4. Revestimiento de la articulación con la fascia temporal o aplicación del disco articular nativo.

5. Reconstrucción de la unidad condílea, ya sea con distracción osteogénica o injerto costocondral y fijación rígida.

6. Movilidad temprana de la mandíbula.

7. Fisioterapia agresiva.

González AGR ${ }^{11}$ menciona que liberar la anquilosis de la articulación no es suficiente para la mejoría del paciente, por lo que para lograr una adecuada función se deberá aumentar la dimensión mediante distracción 
o injerto. De ahí que, en el presente caso, se realizó la eliminación amplia de la masa anquilosada y reconstrucción, seguida de injerto costocondral.

\section{BIBLIOGRAFÍA}

1. Kaban L, Bouchard C, Troulis MA. Protocol for management of temporomandibular joint ankylosis in children. J Oral Maxillofac Surg. 2009; 67: 1966-1978.

2. Kaban L, Perrot DH, Fisher K. A protocol for management of temporomandibular joint ankylosis. J Oral Maxillofac Surg. 1990; 48(11): 1145-1151.

3. Molina D, Aguayo P, Ulloa C, Iturriaga V, Bornhardt T, Saavedra M. Anquilosis de la articulación temporomandibular: una revisión de la literatura. Av Odontoestomatol. 2013; 29 (5): 239-244.

4. Movahed R, Mercuri LG. Management of temporomandibular joint ankylosis. Oral Maxillofac Surg Clin North Am. 2015; 27 : 27-35.

5. González ME, Hernández CA. Anquilosis temporomandibular. Revisión de la literatura. Protocolo de manejo e informe de un caso. Rev ADM. 2007; 64 (6): 238-243.

6. Loreto JGR, Noriega JCL, Rodriguez RR. Anquilosis temporomandibular. Liberación, reconstrucción con injerto de apófisis coronoides y cartílago articular: presentación de caso clínico. Rev Odont Mex. 2011; 15 (2): 115-121.

7. Aparicio LG, Parri FJ, Sancho MA, Sarget R, Morales L. Anquilosis de la articulación temporomandibular (ATM) en la edad pediátrica. Cir Pediatr. 2000; 13: 62-63.

8. Sawhney CP. Bony ankylosis of the temporomandibular joint: follow-up of 70 patients treated with arthroplasty and acrylic spacer interposition. Plast Reconstr Surg. 1986; 77(1): 29-40.

9. Belmont-Laguna F, Sanchez Matus L, Tellez-Rodriguez J, Ceballos Hernandez $\mathrm{H}$. Terapia Funcional en el postoperatorio de la anquilosis temporomandibular en pacientes pediátricos (1 $1^{\mathrm{a}}$ parte). Acta Pediatr Mex. 2007; 28(3): 111-117.

10. Mercado F. Tratamiento de anquilosis temporomandibular con materiales aloplásticos en niños. Reporte de tres casos. Rev ADM. 2018; 75 (1): 55-60.

11. González AGR. Reconstrucción de la articulación temporomandibular: injertos autólogos. Rev Esp Cir Oral Maxilofac. 2005; 27(1): 15-28.

12. Prasad C, Uma Maheswari G, Karthikeyan D. Fate of costochondral graft in temporomandibular joint reconstruction: a histological study. J Maxillofac Oral Sur. 2015; 15(2): 179-183.

13. Arevalo $\mathrm{RH}$, Hurtado JAH, Frias MAN, Medlich MAM. Reconstrucción mandibular con injerto costocondral en microsomía hemifacial. Reporte de un caso. Rev ADM. 2015; 72(4): 212-217. 